\title{
Stratified epithelium in prostatic adenocarcinoma: a mimic of high-grade prostatic intraepithelial neoplasia
}

\author{
Omar Hameed* and Peter A Humphrey \\ Department of Pathology and Immunology, Washington University Medical Center, St Louis, MO, USA
}

\begin{abstract}
Typically glands of prostatic adenocarcinoma have a single cell lining, although stratification can be seen in invasive carcinomas with a cribriform architecture, including ductal carcinoma. The presence and diagnostic significance of stratified cells within non-cribriform carcinomatous prostatic glands has not been well addressed. The histomorphological features and immunohistochemical profile of cases of non-cribriform prostatic adenocarcinoma with stratified malignant glandular epithelium were analyzed. These cases were identified from needle biopsy cases from the consultation files of one of the authors and from a review of 150 consecutive in-house needle biopsy cases of prostatic adenocarcinoma. Immunohistochemistry was performed utilizing antibodies reactive against high molecular weight cytokeratin (34 $\beta E 12)$, p63 and $\alpha$-methylacylcoenzyme-A racemase (AMACR). A total of 8 cases were identified, including 2 from the 150 consecutive inhouse cases (1.3\%). In 4 cases, the focus with glands having stratified epithelium was the sole carcinomatous component in the biopsy, while such a component represented $5-30 \%$ of the invasive carcinoma seen elsewhere in the remaining cases. The main attribute in all these foci was the presence of glandular profiles lined by several layers of epithelial cells with cytological and architectural features resembling flat or tufted high-grade prostatic intraepithelial neoplasia, but lacking basal cells as confirmed by negative 34/E12 and/or p63 immunostains in all cases. The AMACR staining profile of the stratified foci was variable, with 4 foci showing positivity, and 3 foci being negative, including two cases that displayed AMACR positivity in adjacent non-stratified prostatic adenocarcinoma. Prostatic adenocarcinoma with stratified malignant glandular epithelium can be identified in prostate needle biopsy samples harboring non-cribriform prostatic adenocarcinoma and resembles glands with high-grade prostatic intraepithelial neoplasia. These 'PIN-like' carcinomas can present in pure form. Recognition of this pattern of prostatic adenocarcinoma is necessary to correctly diagnose such cases as invasive carcinoma.
\end{abstract}

Modern Pathology (2006) 19, 899-906. doi:10.1038/modpathol.3800601; published online 7 April 2006

Keywords: prostate; carcinoma; prostatic intraepithelial neoplasia; stratification

High-grade prostatic intraepithelial neoplasia (HGPIN) and invasive prostatic adenocarcinoma are neoplastic proliferations with similar, but not identical, histochemical, immunophenotypic, and genotypic features, ${ }^{1-7}$ as well as gene expression profiles. ${ }^{8}$ In addition, HG-PIN, especially cribriform and flat HG-PIN, can mimic invasive prostatic adenocarcinoma and immunohistochemistry may

Correspondence: Dr PA Humphrey, MD, PhD, Department of Pathology and Immunology, Washington University Medical Center, 660 South Euclid Avenue, Campus Box 8118, St Louis, MO 63110, USA.

E-mail: humphrey@path.wustl.edu

${ }^{*}$ Current address: Department of Pathology, University of Alabama at Birmingham, Birmingham, Alabama

Received 30 November 2005; revised 8 March 2006; accepted 9 March 2006; published online 7 April 2006 be needed to document the presence of basal cells in the former. ${ }^{9} \mathrm{We}^{6}$ and others ${ }^{10,11}$ have previously noticed stratified epithelium in prostatic adenocarcinoma, where the converse can occur; prostatic adenocarcinoma mimicking non-cribriform HG-PIN. This histomorphologic presentation of prostatic adenocarcinoma has not been well addressed in the literature. We recently encountered several prostate needle biopsy cases in consultation that showed stratified epithelium within non-cribriform prostatic adenocarcinoma glands, including some, where the diagnosis of invasive carcinoma was questioned. The aim of this investigation was to characterize the frequency, as well as the histological and immunophenotypic attributes of prostatic adenocarcinomas with this appearance and report the features of these 'PIN-like' carcinomas which distinguish them from HG-PIN. 


\section{Materials and methods}

We analyzed the morphological features and immunohistochemical profile of cases of prostatic adenocarcinoma showing stratified epithelium within carcinomatous glands, as seen in prostate needle biopsies. These cases were prospectively identified in formalin-fixed, paraffin-embedded prostate needle biopsy samples presented to one of the authors in consultation over a 12-month period (November 2004 to October 2005), as well as from a review of 150 consecutive in-house needle biopsy cases of prostatic adenocarcinoma (diagnosed between 1996 and 1998), identified via computer search of the database files of the Lauren V Ackerman Laboratory of Surgical Pathology, Washington University Medical Center. Any degree of stratification was sufficient for inclusion in the study, while cases displaying a cribriform architecture were excluded. The clinical presentation of the patients was obtained from the surgical pathology reports and/or the patients' medical charts. Review of the H\&E sections was performed to assign a Gleason grade, to estimate the extent of carcinoma (by measuring both the linear extent of carcinoma and the percentage of core tissue involvement as previously defined, ${ }^{12,13}$ ) as well as to measure the percentage of carcinomatous glands displaying stratified epithelium in each case. Immunohistochemistry was performed utilizing either a prediluted p63/ $\alpha$-methylacyl-coenzyme-A racemase (AMACR) antibody cocktail or one prepared in our laboratory (prediluted: 'PIN Cocktail', BC4A4/13H4 clones, Biocare Medical, Walnut Creek, CA; homemade: BC4A4/13H4 clones, Neomarkers, Fremont, CA and Zeta Corporation, Sierra Madre, CA, respectively, $1 / 500$ dilution for both) for all cases, as well as the $34 \beta$ E12 antibody (clone $34 \beta E 12$, Dakocytomation, Carpinteria, CA, 1/100) for a subset of the cases.
Heat-induced antigen retrieval was performed for p63 and AMACR immunohistochemistry by placing the sections in EDTA buffer ( $\mathrm{pH} 8$ ) in a Decloaking chamber (Biocare Medical) for $3 \mathrm{~min}$, while proteinase $\mathrm{K}$ incubation at room temperature for $5 \mathrm{~min}$ was used for $34 \beta \mathrm{E} 12$. The pattern of immunohistochemical reactivity of the foci of prostatic adenocarcinoma glands with stratified epithelium was evaluated and compared to that in non-stratified malignant acini. Immunohistochemistry was either performed during the original evaluation of the case where malignancy was questioned, or after case identification for the purposes of this study.

\section{Results}

A total of eight cases were identified, six consult cases and two from consecutive in-house cases, representing $1 \%$ of prostatic adenocarcinomas diagnosed on needle biopsy. The clinical and pathological features of these cases are presented in Table 1. The age of the patients ranged from 61 to 89 years (mean, 73 years; median, 72 years). The serum prostate-specific antigen (PSA) level was known to be elevated in six patients, ranging from 4.3 to $66.2 \mathrm{ng} / \mathrm{ml}$ (mean, $18.2 \mathrm{ng} / \mathrm{ml}$; median, $5.5 \mathrm{ng} /$ $\mathrm{ml}$ ) and three patients were also known to have prostatic nodules on digital rectal examination. The extent of carcinoma in these cases ranged from $<1$ (minimal prostatic adenocarcinoma; ${ }^{14}$ two cases) to $26 \mathrm{~mm}$ (mean, $10.2 \mathrm{~mm}$; median, $7 \mathrm{~mm}$ ), representing $<5$ up to $75 \%$ of the needle biopsy tissues. In four cases (cases 2, 3, 5, and 6), the foci with glands having stratified epithelium were the sole carcinomatous components in the biopsies, while such a component represented $5-30 \%$ of the invasive prostatic adenocarcinoma seen elsewhere in the tissue cores of the remaining cases. The main

Table 1 Clinical and pathological findings of eight cases of prostatic adenocarcinoma with stratified malignant glandular epithelium

\begin{tabular}{|c|c|c|c|c|c|c|c|c|c|c|}
\hline \multirow[t]{3}{*}{ Case no. } & \multirow[t]{3}{*}{ Age } & \multirow{3}{*}{$\begin{array}{l}\text { PSA level } \\
\text { (ng/ml) }\end{array}$} & \multicolumn{3}{|c|}{ Light microscopic findings } & \multicolumn{5}{|c|}{ Immunohistochemical findings } \\
\hline & & & \multicolumn{2}{|c|}{ Carcinoma extent } & \multirow[t]{2}{*}{ Grade } & \multirow{2}{*}{$\begin{array}{c}\% \\
\text { carcinoma } \\
\text { stratified }\end{array}$} & \multirow[t]{2}{*}{$34 \beta E 12$} & \multirow[t]{2}{*}{ p63 } & \multicolumn{2}{|c|}{$A M A C R$} \\
\hline & & & $\begin{array}{l}\text { Length } \\
\text { (in } \mathrm{mm} \text { ) }\end{array}$ & $\begin{array}{l}\% \text { of } \\
\text { tissue }\end{array}$ & & & & & Stratified & $\begin{array}{c}\text { Non- } \\
\text { stratified }\end{array}$ \\
\hline 1 & 61 & 4.6 & 11 & 45 & $3+3$ & 30 & Negative & Negative & Positive & Positive \\
\hline $2^{\mathrm{a}}$ & 89 & 66.2 & 17 & 55 & $3+3$ & 100 & Negative & Negative & Positive & NA \\
\hline $3^{a}$ & 71 & $\mathrm{U}$ & 1.5 & $<5$ & $3+3$ & 100 & Negative & $\mathrm{NC}$ & $\mathrm{NC}$ & NA \\
\hline 4 & 68 & 23.0 & 21 & 75 & $3+4^{\mathrm{b}}$ & 5 & - & Negative & Negative & Positive \\
\hline $5^{a}$ & 73 & 5.8 & $<1$ & $<5$ & $3+3$ & 100 & Negative & Negative & Negative & NA \\
\hline $6^{\mathrm{a}}$ & 64 & 4.3 & $<1$ & $<5$ & $3+3$ & 100 & - & Negative & Positive & NA \\
\hline 7 & 74 & 5.2 & 3 & 10 & $3+3$ & 15 & - & Negative & Positive & Positive \\
\hline 8 & 81 & $\mathrm{U}$ & 26 & 70 & $3+4^{b}$ & 10 & - & Negative & Negative & Positive \\
\hline
\end{tabular}

AMACR, $\alpha$-methylacyl-coenzyme-A racemase; PSA, prostate-specific antigen; U, unknown; NC, non-contributory (focus no longer present on section); NA, not applicable.

${ }^{\mathrm{a}}$ Immunohistochemistry was performed during original evaluation of the case.

${ }^{\mathrm{b}}$ Gleason pattern 4 carcinoma was limited to the non-stratified component. 
attribute in all these foci was the presence of single, separate glandular profiles (Gleason grade 3) lined by several layers of epithelial cells with cytological and architectural features resembling flat or tufted HG-PIN (Figures 1-5). They differed from HG-PIN by showing a greater degree of glandular crowding and/or disorder than is usually seen with glands involved by HG-PIN. The malignant epithelial cells in the stratified glands were cuboidal or columnar in shape, with rounded or oval to fusiform nuclei, and the cytological features were very similar to those seen in the non-stratified prostatic adenocarcinoma component, when present. Both the stratified and non-stratified foci in all cases lacked basal cells, which was confirmed by a negative immunohistochemical reaction for p63 in seven cases, and/or a negative $34 \beta \mathrm{E} 12$ immunostain in the four cases that were examined with this antibody. The AMACR staining profile of the stratified foci was variable, with four foci showing positivity, a profile similar to the adjacent prostatic adenocarcinoma without stratification when present, and three foci being negative, including two cases that displayed AMACR positivity in the adjacent non-stratified prostatic adenocarcinoma.

\section{Discussion}

In this study, we have found that stratified epithelium in non-cribriform glands of prostatic adenocarcinoma can be identified in prostate needle biopsy specimens with prostatic adenocarcinoma and can resemble HG-PIN glands. Carcinomas with PIN-like features appear to be uncommon as they accounted for only $1.3 \%$ of consecutive prostatic adenocarcinomas diagnosed over a 2-year period. Although these PIN-like carcinomas were only AMACR positive in $50 \%$ of cases, they are invariably negative for basal cell markers, a finding that is of utility in evaluating crowded, stratified glands with cytological atypia, where PIN-like prostatic
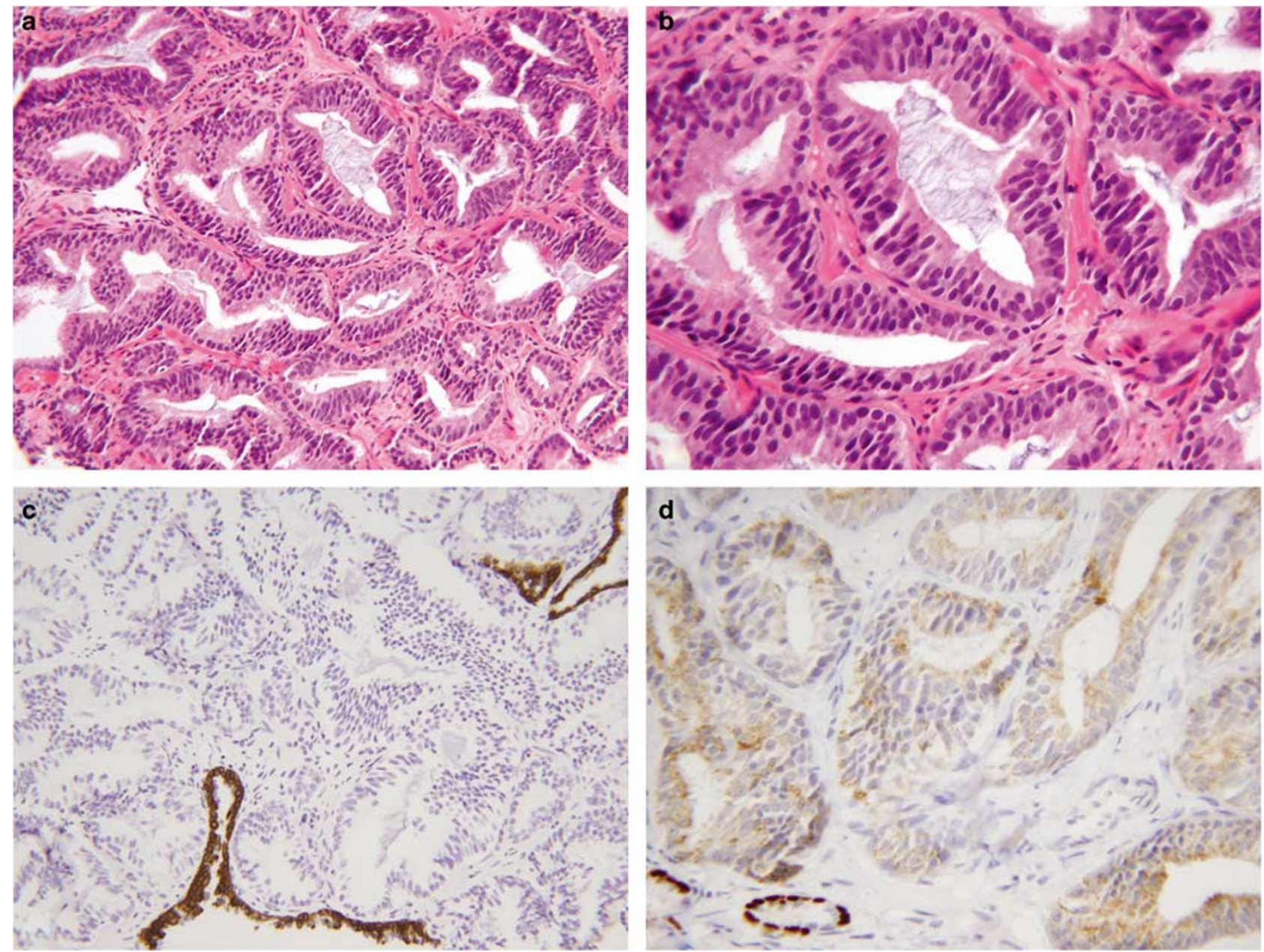

Figure 1 A case (no. 1) of prostatic adenocarcinoma composed of small to medium-sized glands lined by stratified epithelium resembling HG-PIN. The degree of glandular crowding is more than what one usually sees with PIN. The malignant glands lack a basal cell lining (a, b) as confirmed by a negative 34ßE12 immunostain (c) as well as a negative p63 immunostain, as demonstrated by a p63/AMACR antibody cocktail (d) that also demonstrates AMACR positivity in these glands, a profile similar to the non-stratified component of the tumor seen elsewhere on the slide (not shown). 
902
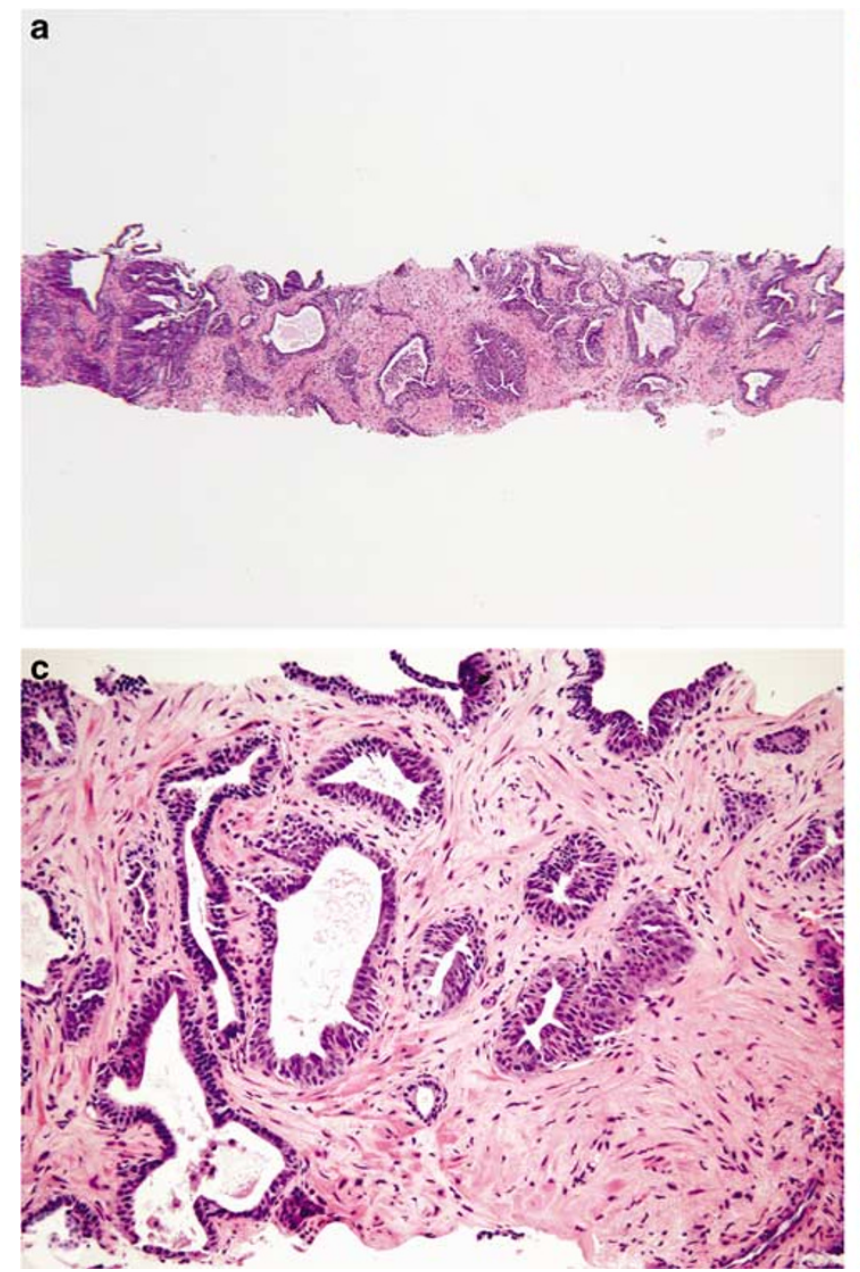
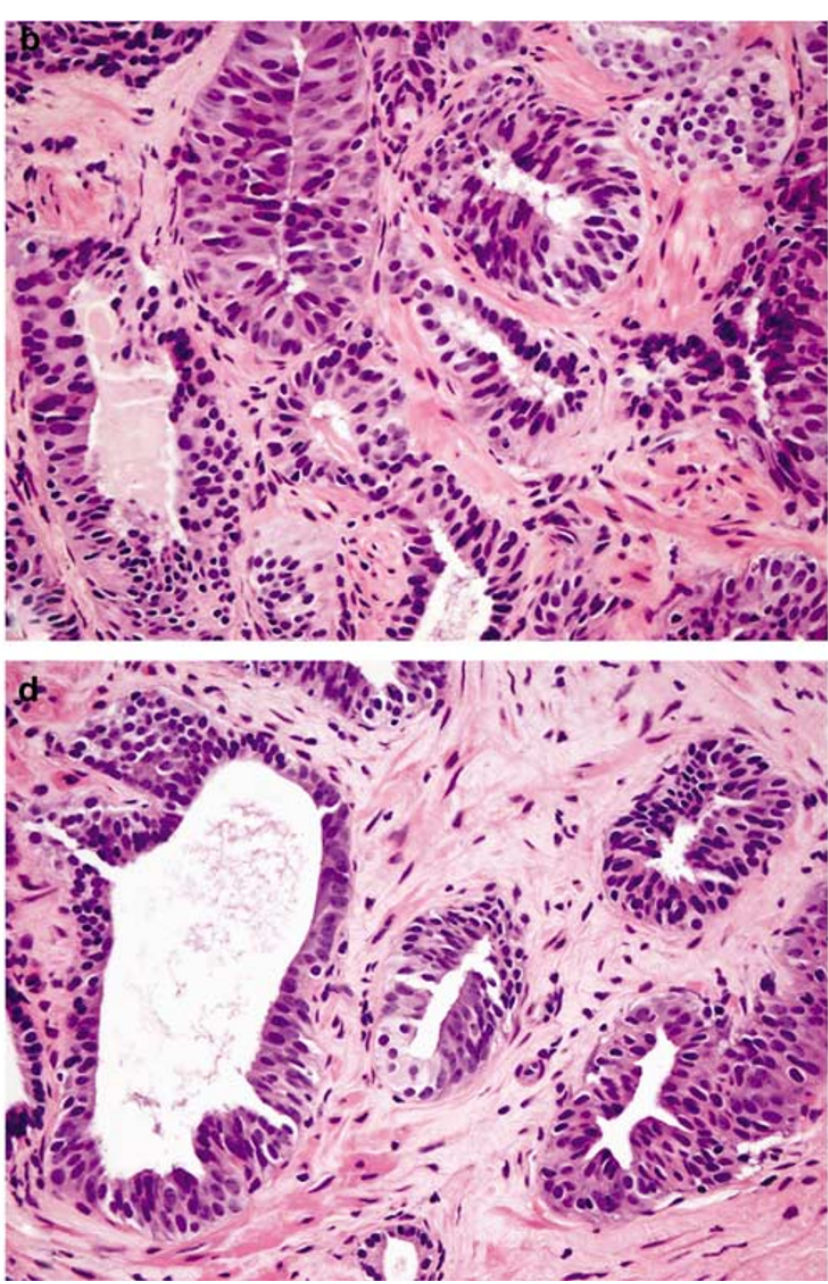

Figure 2 Another case (no. 2) of 'PIN-like' prostatic adenocarcinoma spanning almost the entire length of the prostate needle biopsy tissue core (a). The tumor displays areas of crowded small-sized stratified malignant glands (b) as well as some that are more dispersed in the stroma, including a few larger dilated glands (c, d). The diagnosis of carcinoma was confirmed by negative $34 \beta \mathrm{E} 12$ and p63 immunostains, as well as AMACR immunoreactivity (not shown).

adenocarcinoma could be in the differential diagnosis in addition to HG-PIN.

Although Gleason's original depiction of the different histological patterns of prostatic adenocarcinoma included an image of a malignant acinus with apparent micropapillary architecture and multilayered epithelium, ${ }^{15}$ most cases of Gleason patterns 1-3 acinar prostatic adenocarcinoma are composed of small acini lined by malignant epithelium in a single cell layer. An exception would be the occasional Gleason pattern 3 carcinomas with a cribriform architecture ${ }^{16}$ which, similar to Gleason pattern 4 carcinomas, can show multilayered epithelium. In addition, although characterized by larger glandular structures, ductal prostatic adenocarcinoma is often seen to have a papillary or cribriform growth pattern with multilayered epithelium. As these cribriform carcinomas, acinar or ductal, resemble cribriform HG-PIN, it is important to be able to distinguish between the two. ${ }^{9,17-19}$ Similarly, several recently described variants of acinar prostatic adenocarcinoma, including foamy gland, ${ }^{20,21}$ pseudohyperplastic, ${ }^{22,23}$ and atrophic ${ }^{24-26}$ variants often have cytological or architectural features that do not readily suggest malignancy, and accordingly may be mistakenly diagnosed as benign especially in prostate needle biopsy specimens if one is unfamiliar with their appearances. As the presence of stratified epithelium in prostatic adenocarcinoma has not been well addressed in the literature and could also be potentially confused with HG-PIN, as was the case in several prostate needle biopsy cases we saw in consultation, we sought to characterize this cellular growth arrangement in prostatic adenocarcinoma.

In this study, we characterize another architectural variation in prostatic adenocarcinoma that could be confused with non-invasive disease: epithelial stratification in non-cribriform malignant glands of prostatic adenocarcinoma. Such an occurrence has been previously noted in radical prostatectomy specimens both within otherwise regular acinar prostatic adenocarcinoma, ${ }^{6,11}$ as well as representing a pattern of perineural invasion of prostatic adeno- 

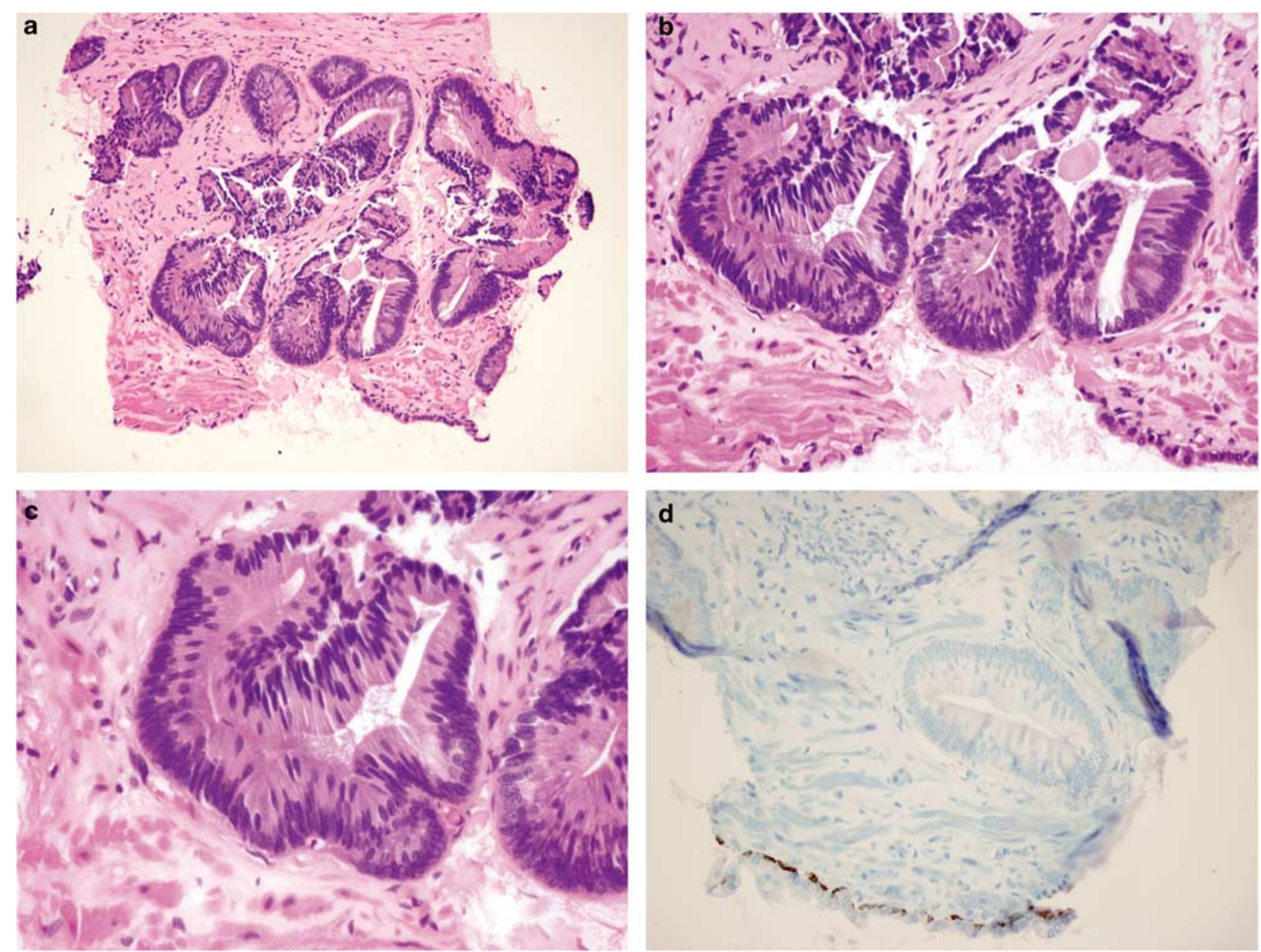

Figure 3 A case (no. 3) of prostatic adenocarcinoma, where small and medium-sized stratified malignant glands are seen streaming across the width of the needle biopsy (a). The nuclei of epithelial cells lining these glands are more elongated than those seen in cases 1 and $2(\mathbf{b}, \mathbf{c})$. A $34 \beta$ E12 immunostain (d) confirms lack of basal cells in this proliferation.

carcinoma; ${ }^{10}$ however, a detailed analysis of this histological variation in prostatic adenocarcinoma, especially in prostate needle biopsy samples, has not been previously addressed in the literature.

We identified prostatic adenocarcinoma with stratified malignant glandular epithelium in six consultation cases submitted over a 12-month period. To assess the incidence of this histological variation, we retrospectively reviewed 150 consecutive nonreferral in-house cases of prostatic adenocarcinoma and identified two additional cases, indicating that the incidence of finding stratified malignant glandular epithelium in prostatic adenocarcinoma was $1.3 \%$. Despite its uncommon frequency, diagnostic recognition of this variation in prostatic adenocarcinoma is important owing to the fact that it closely resembles glands involved by flat or tufted HG-PIN which, if found on biopsy, would typically result in a non-therapeutic clinical approach in the management for the patient (follow-up), as opposed to the active therapy often undertaken following a diagnosis of invasive adenocarcinoma. Of note, our observations are similar to those of Amin et al, ${ }^{10}$ who found foci of prostatic adenocarcinoma around nerves that very much resembled micropapillary HG-PIN which they termed 'circumferential micropapillary perineural invasion' and argued that they could also be misdiagnosed as HG-PIN.

The fact that stratified malignant glandular epithelium was the sole histological appearance of malignancy in one-half of our cases also highlights the importance of recognizing this feature in prostatic adenocarcinoma. In the other cases, stratified malignant glandular epithelium represented $5-30 \%$ of the malignant glands in our cases of prostatic adenocarcinoma. The finding of identical cytological features in the malignant epithelial cells in both stratified and non-stratified glands (when present) argues that stratification in prostatic adenocarcinoma is more likely to represent an unusual growth pattern rather than a specific variant of prostatic adenocarcinoma.

The main differential diagnosis of prostatic adenocarcinoma with stratified malignant glandular epithelium in needle biopsy samples centers on HG-PIN. This distinction might not be critical if 

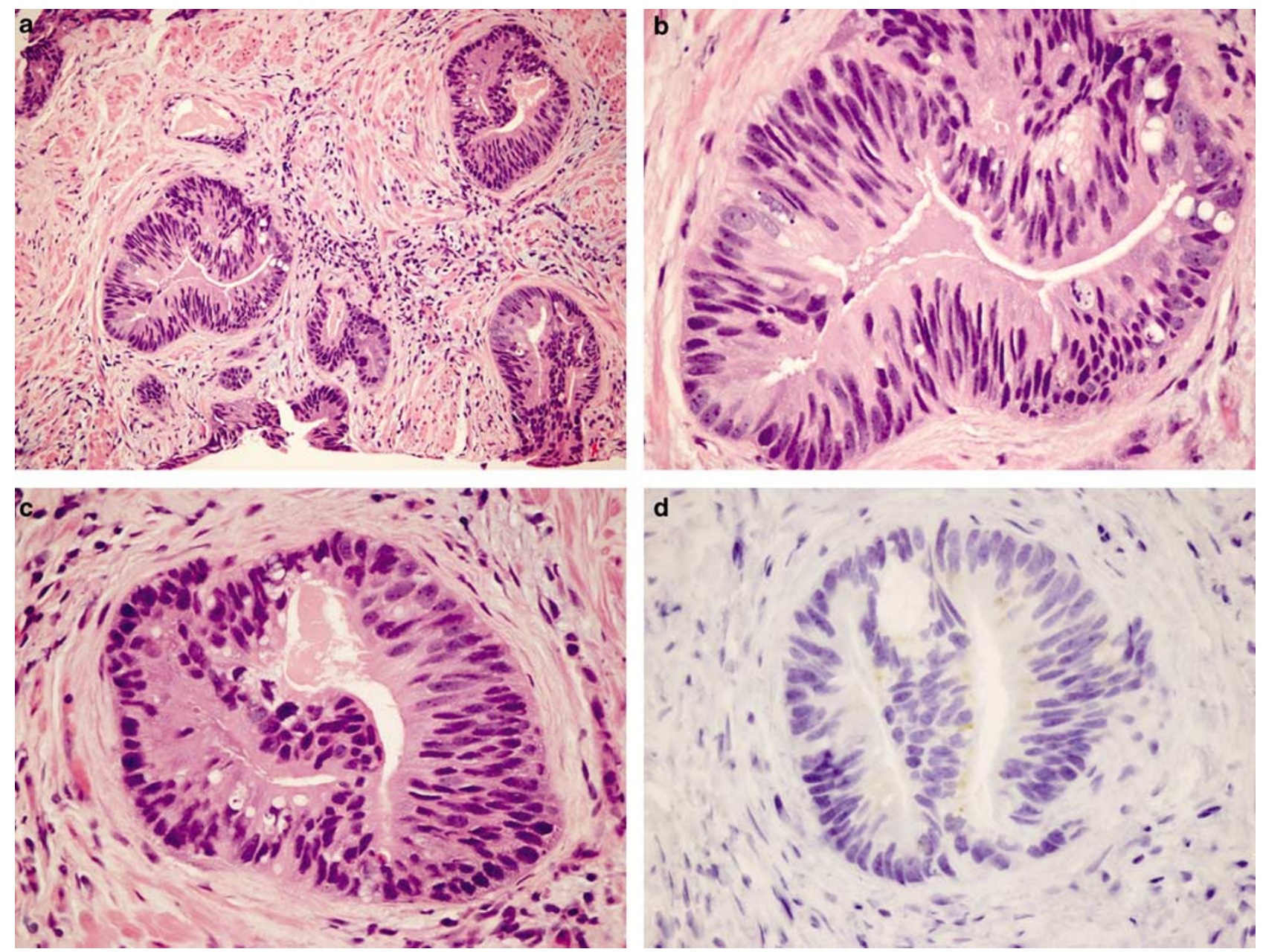

Figure 4 A case (no. 4) of prostatic adenocarcinoma, where the malignant glands are not crowded and appear dispersed in the prostatic stroma (a). The glands show prominent epithelial stratification as well as elongated nuclei (b, c). A p63/AMACR antibody cocktail immunostain (d) confirms lack of basal cells and shows only focal and minimal granular cytoplasmic staining which would be considered insufficient for AMACR positivity.

areas of unequivocal conventional prostatic adenocarcinoma are identified elsewhere in the biopsy, but is definitely so if no such other areas exist. The identification of basal cells in H\&E sections or by basal cell marker positivity in immunohisto chemistry would point towards a diagnosis of HGPIN, whereas lack of basal cells and the presence of glandular crowding would point towards a diagnosis of prostatic adenocarcinoma. AMACR immunostaining has no role in this distinction as it cannot differentiate between prostatic adenocarcinoma and HG-PIN. ${ }^{9,27}$ Another diagnostic consideration is ductal adenocarcinoma, which although usually characterized by malignant papillary or cribriform glands lined by pseudostratified columnar cells, ${ }^{6,28}$ has been reported to occasionally infiltrate as single glands lined by pseudostratified columnar epithelium resembling infiltrating colonic adenocarcinoma. ${ }^{29,30}$ In fact, three of the cases of PIN-like carcinomas reported herein (cases 3, 4, and 5) bear a certain degree of morphologic resemblance to published images ${ }^{29,30}$ of this pattern of ductal adenocarcinoma, and may very well be examples of this form of adenocarcinoma. However, it should be noted that in the other five cases the stratified malignant epithelium was composed of non-columnar cells with rounded nuclei, quite similar to what may be seen in ordinary acinar adenocarcinoma. As there are no specific markers available that can reliably distinguish between ductal and acinar carcinoma, it is difficult to determine with certainty the acinar vs ductal differentiation status of our cases, but based on our findings, it does appear that stratified malignant glands are not limited to a specific type of prostatic adenocarcinoma. The final differential diagnostic consideration for PIN-like carcinoma, especially those with columnar cells and elongated nuclei, is metastatic colonic carcinoma, which may or may not be suggested by the clinical history. In these situations, immunohistochemical reactivity with PSA (and/or prostatic acid phosphatase) would point to a prostatic primary, while CDX2 or villin immunoreactivity would point to a colonic primary. Immunostains for cytokeratins 

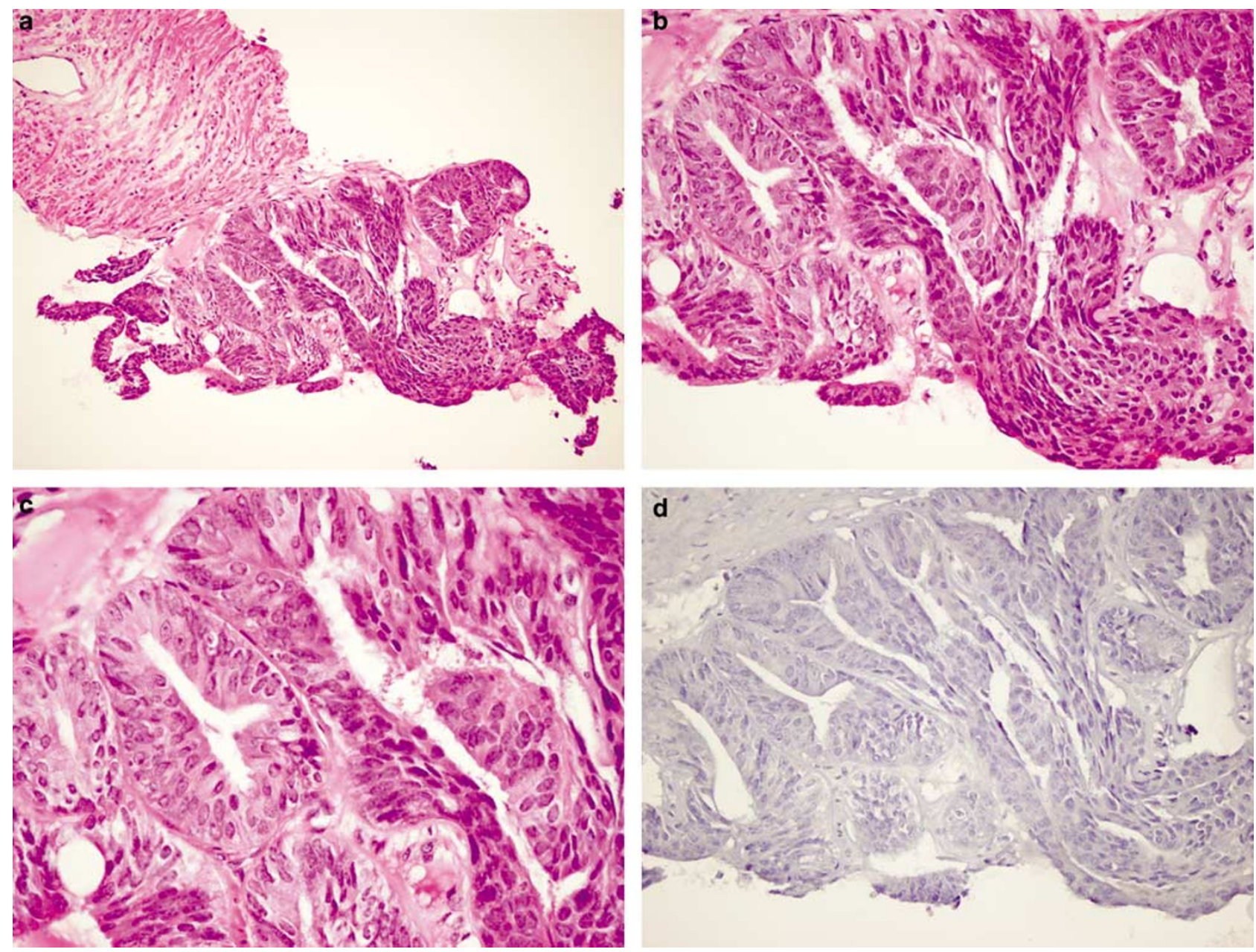

Figure 5 A case (no. 5) of prostatic adenocarcinoma composed of crowded medium-sized malignant glands with prominent epithelial stratification (a-c). No basal cells were identified on the hematoxylin and eosin-stained sections, with a $34 \beta$ E12 immunostain (not shown), or with a p63/AMACR antibody cocktail immunostain (d). The proliferation is also negative for AMACR (d).

7 and 20 are less useful in this regard because of significant overlap in expression. ${ }^{9}$

One limitation of this study is the lack of radical prostatectomy and clinical follow-up information for the cases presented here, which might have further delineated the 'histologic lineage' of PIN-like carcinoma as well as identified any prognostic implications of this growth pattern of prostatic adenocarcinoma. The main finding of our investigation is that diagnostic recognition of this pattern of growth in prostatic adenocarcinoma is important to avoid underdiagnosis of invasive carcinoma.

In summary, we have found that non-cribriform prostatic adenocarcinomas in prostate needle biopsy samples can be composed of glands lined by stratified malignant glandular epithelium and can resemble glands involved by HG-PIN. These PINlike carcinomas can present in pure form. Diagnostic recognition of this unusual growth variation of prostatic adenocarcinoma is critical to avoid underdiagnosis of invasive carcinoma in prostate needle biopsy samples.

\section{References}

1 Baltaci S, Orhan D, Ozer G, et al. Bcl-2 proto-oncogene expression in low- and high-grade prostatic intraepithelial neoplasia. BJU Int 2000;85:155-159.

2 Bostwick DG, Pacelli A, Lopez-Beltran A. Molecular biology of prostatic intraepithelial neoplasia. Prostate 1996;29:117-134.

3 Emmert-Buck MR, Vocke CD, Pozzatti RO, et al. Allelic loss on chromosome 8p12-21 in microdissected prostatic intraepithelial neoplasia. Cancer Res 1995;55: 2959-2962.

4 Qian J, Bostwick DG, Takahashi S, et al. Chromosomal anomalies in prostatic intraepithelial neoplasia and carcinoma detected by fluorescence in situ hybridization. Cancer Res 1995;55:5408-5414.

5 Willman JH, Holden JA. Immunohistochemical staining for DNA topoisomerase II-alpha in benign, premalignant, and malignant lesions of the prostate. Prostate 2000;42:280-286.

6 Humphrey PA. Prostate Pathology. American Society for Clinical Pathology: Chicago, 2003, 556pp.

7 Hameed O, Humphrey PA. p63/AMACR antibody cocktail restaining of prostate needle biopsy tissues 
after transfer to charged slides: a viable approach in the diagnosis of small atypical foci that are lost on block sectioning. Am J Clin Pathol 2005;124:708-715.

8 Ashida S, Nakagawa H, Katagiri T, et al. Molecular features of the transition from prostatic intraepithelial neoplasia (PIN) to prostate cancer: genome-wide geneexpression profiles of prostate cancers and PINs. Cancer Res 2004;64:5963-5972.

9 Hameed O, Humphrey P. Immunohistochemistry in diagnostic surgical pathology of the prostate. Semin Diagn Pathol 2005;22:88-104.

10 Amin MB, Cabrera RA, Lim SD, et al. All that is micropapillary is not high-grade prostatic intraepithelial neoplasia (HG-PIN): Circumferential micropapillary perineural invasion (CMPNI) - a potential pitfall in the recognition of invasive prostatic adenocarcinoma (abstract). Mod Pathol 2003;15:139A.

11 Bostwick DG, Dundore PA. Figure 8.5a in Biopsy Pathology of the Prostate, 1st edn. Chapman \& Hall: New York, 1997, 267pp.

12 Bismar TA, Lewis Jr JS, Vollmer RT, et al. Multiple measures of carcinoma extent versus perineural invasion in prostate needle biopsy tissue in prediction of pathologic stage in a screening population. Am J Surg Pathol 2003;27:432-440.

13 Lewis Jr JS, Vollmer RT, Humphrey PA. Carcinoma extent in prostate needle biopsy tissue in the prediction of whole gland tumor volume in a screening population. Am J Clin Pathol 2002;118:442-450.

14 Thorson P, Humphrey PA. Minimal adenocarcinoma in prostate needle biopsy tissue. Am J Clin Pathol 2000; 114:896-909.

15 Gleason DF. Histologic grading of prostatic carcinoma. In: Bostwick DG (ed). Pathology of the Prostate. Churchill Livingstone: New York, 1990, pp 83-93.

16 Epstein JI, Allsbrook WCJ, Amin MB, et al. The 2005 International Society of Urological Pathology (ISUP) consensus conference on Gleason grading of prostatic carcinoma. Am J Surg Pathol 2005;29:1228-1242.

17 Srigley JR. Benign mimickers of prostatic adenocarcinoma. Mod Pathol 2004;17:328-348.

18 Amin MB, Schultz DS, Zarbo RJ. Analysis of cribriform morphology in prostatic neoplasia using antibody to high-molecular-weight cytokeratins. Arch Pathol Lab Med 1994;118:260-264.
19 Kronz JD, Shaikh AA, Epstein JI. Atypical cribriform lesions on prostate biopsy. Am J Surg Pathol 2001;25: 147-155.

20 Nelson RS, Epstein JI. Prostatic carcinoma with abundant xanthomatous cytoplasm. Foamy gland carcinoma. Am J Surg Pathol 1996;20:419-426.

21 Tran TT, Sengupta E, Yang XJ. Prostatic foamy gland carcinoma with aggressive behavior: clinicopathologic, immunohistochemical, and ultrastructural analysis. Am J Surg Pathol 2001;25:618-623.

22 Humphrey PA, Kaleem Z, Swanson PE, et al. Pseudohyperplastic prostatic adenocarcinoma. Am J Surg Pathol 1998;22:1239-1246.

23 Levi AW, Epstein JI. Pseudohyperplastic prostatic adenocarcinoma on needle biopsy and simple prostatectomy. Am J Surg Pathol 2000;24:1039-1046.

24 Cina SJ, Epstein JI. Adenocarcinoma of the prostate with atrophic features. Am J Surg Pathol 1997;21:289-295.

25 Egan AJ, Lopez-Beltran A, Bostwick DG. Prostatic adenocarcinoma with atrophic features: malignancy mimicking a benign process. Am J Surg Pathol 1997; 21:931-935.

26 Kaleem Z, Swanson PE, Vollmer RT, et al. Prostatic adenocarcinoma with atrophic features: a study of 202 consecutive completely embedded radical prostatectomy specimens. Am J Clin Pathol 1998;109:695-703.

27 Hameed O, Sublett J, Humphrey PA. Immunohistochemical stains for p63 and alpha-methylacyl-CoA racemase, versus a cocktail comprising both, in the diagnosis of prostatic carcinoma: a comparison of the immunohistochemical staining of 430 foci in radical prostatectomy and needle biopsy tissues. Am J Surg Pathol 2005;29:579-587.

28 Yang XJ, Cheng L, Helpap B, et al. Ductal adenocarcinoma. In: Eble JN, Sauter G, Epstein JI, Sesterhenn IA (eds). Pathology and Genetics of Tumours of the Urinary System and Male Genital Organs. IARC Press: Lyon, France, 2004, pp 199-201.

29 Epstein JI, Yang XJ. Figure 11.11 in Prostate Biopsy Interpretation, 3nd edn. Lippincott Williams \& Wilkins: Philadelphia, 2002, 304pp.

30 Epstein JI, Woodruff JM. Adenocarcinoma of the prostate with endometrioid features. A light microscopic and immunohistochemical study of ten cases. Cancer 1986;57:111-119. 